\title{
Épidémiologie des parasitoses digestives des jeunes bovins dans le Nord-Cameroun
}

\author{
J.-Y. Chollet ${ }^{1 *}$, A. Martrenchar ${ }^{2 * *}$, D. Bouchel ${ }^{2 * *}$, A. Njoya ${ }^{2}$
}

CHOLLET (J.-Y.), MARTRENCHAR (A.), BOUCHEL (D.), NJOYA (A.). Épidémiologie des parasitoses digestives des jeunes bovins dans le Nord-Cameroun. Revue Élev. Méd. vét. Pays trop., 1994, 47 (4) : 365-374

Les parasitoses digestives des jeunes bovins zébus en milieu traditionnel du Nord-Cameroun ont été étudiées grâce à un suivi coproscopique mensuel de 17 troupeaux pendant 2 ans. La toxocarose apparaît comme la plus importante dans la province du Nord, où sa prévalence atteint 58 p. 100 entre 0 et 6 mois ; 60 p. 100 des veaux positifs excrètent au moins une fois de grandes quantités d'œufs de Toxocara; la fréquence des prélèvements révélant des nombres d'ocufs élevés s'est avéréc plus grande en saison sèche. La vermifugation des veaux à un mois contre les Toxocara avec un produit bon marché pourrait être rentable dans le Nord. La strongyloïdose atteint 75,5 p. 100 des veaux de 0 à 12 mois, les nombres d'œufs de Strongyloides étant élevés au moins une fois chez 31 p. 100 de ces veaux. Comme pour la toxocarose, les veaux sont plus souvent et davantage parasités dans le Nord que dans l'Extrême-Nord. L'importance clinique de la strongyloïdose semble assez faible, les rares troubles enregistrés se confondant alors avec ceux provoqués par la toxocarose. $L$ 'importance des strongyloses digestives est plus difficile à évaluer : tous les bouvillons sont atteints au cours de leur carrière, et de la même façon dans les deux provinces; les nombres d'oufs de strongles dans les fèces des bovins de plus de six mois sont élevés pour $6,8 \mathrm{p} .100$ des prélèvements, et au moins une fois chez $35 \mathrm{p} .100$ de ces animaux. Ces résultats ne permettent pas de préconiser $a$ priori un programme de vermifugation systématique contre les strongles, plusieurs programmes simples devant être testés pour déterminer leur rentabilité. Des coccidies ont été reconnues sur 77,4 p. 100 des veaux de 0 à 12 mois, les infections étant plus fréquentes et plus importantes dans le Nord, mais elles sont rarement fortes. Les Trichuris, Moniezia, Fasciola et paramphistomes n'ont été mis en évidence que sur un petit nombre de cas. En ce qui concerne les nématodoses, les interventions curatives et les études "coûts-bénéfices" de programmes de vermifugations doivent donc être ciblées sur la toxocarose des veaux de $\mathbf{0 - 3}$ mois dans le Nord et les strongyloses des jeunes de 6-12 mois dans les 2 provinces.

Mots clés : Bovin - Zébu - Helminthose - Coccidiose - Strongylidae - Tri chostrongylidae - Strongyloides - Toxocara vitulorum Epidémiologie Contrôle de maladies - Analyse coût-bénéfice - Elevage traditionnel Cameroun.

1. Laboratoire national vétérinaire de Boklé, Garoua, B.P. 503, Cameroun.

2. Institut de Recherches zootechniques et vétérinaires, Garoua, B.P. 1073, Cameroun.

* Adresse actuelle : DSV du Gers, 108 Av. de la 1ère Armée française, 32020 Auch, France.

** Adresse actuelle: Mission française de Coopération, 01 B.P 1839, Abidjan 01, Côte d'Ivoire.

*** Adresse actuelle : CIRAD-EMVT, 10 rue Pierre-Curie, 94704 Maisons-Alfort Cedex, France.

Reçu le 24.11.1993, accepté le 24.01. 1995.

\section{INTRODUCTION}

Les études parasitologiques réalisées antérieurement sur les bovins du Nord-Cameroun grâce à du matériel d'abattoir $(6,11)$ ont permis principalement de recenser les différentes espèces parasites des bovins et de déterminer leur fréquence. Cependant, deux phénomènes limitent considérablement l'utilisation de données d'abattoir pour estimer l'impact de maladies endémiques dans cette région :

- les animaux vendus pour la boucherie sont essentiellement des animaux âgés (l'abattage des veaux est d'ailleurs interdit) et en mauvais état, malades et/ou sousalimentés :

- les circuits complexes de commercialisation font qu'une grande partie des animaux abattus dans le Nord-Cameroun proviennent de régions éloignées.

La façon la plus précise d'étudier l'épidémiologie de ces parasitoses digestives eût été d'utiliser des animaux traceurs sacrifiés à intervalles de temps déterminés. Cette méthode nécessite malheureusement des moyens financiers et humains importants. Un suivi coproscopique permet cependant d'évaluer la prévalence réelle des groupes de parasites et, dans la mesure où l'on connaît par ailleurs les espèces parasites les plus fréquentes, leur importance pathologique dans la région.

L'objectif de cette étude était donc d'apprécier la signification pathologique des différentes parasitoses digestives rencontrées afin de mieux cibler les traitements curatifs et d'évaluer l'intérêt potentiel d'interventions systématiques.

\section{MATÉRIEL ET MÉTHODES}

\section{Milieu et caractéristiques des élevages}

Ce travail a été mené sur 17 troupeaux dans 8 localités des provinces du Nord et de l'Extrême-Nord du Cameroun, réparties entre $9^{\circ} 01^{\prime}$ et $10^{\circ} 26^{\prime}$ de latitude nord (carte 1). Les caractéristiques géographiques et les typologies botaniques de ces terroirs sont données dans le tableau I.

Les animaux sont des zébus élevés en milieu traditionnel, excepté pour un troupeau en station zootechnique à Lougguéré (troupeau $\mathrm{n}^{\circ} 20$ ). Les types génétiques sont variés : zébus Goudali, Bokolo, Mbororo (Red Fulani), Akou (White Fulani), Arabes Choa, et leurs croisements. 


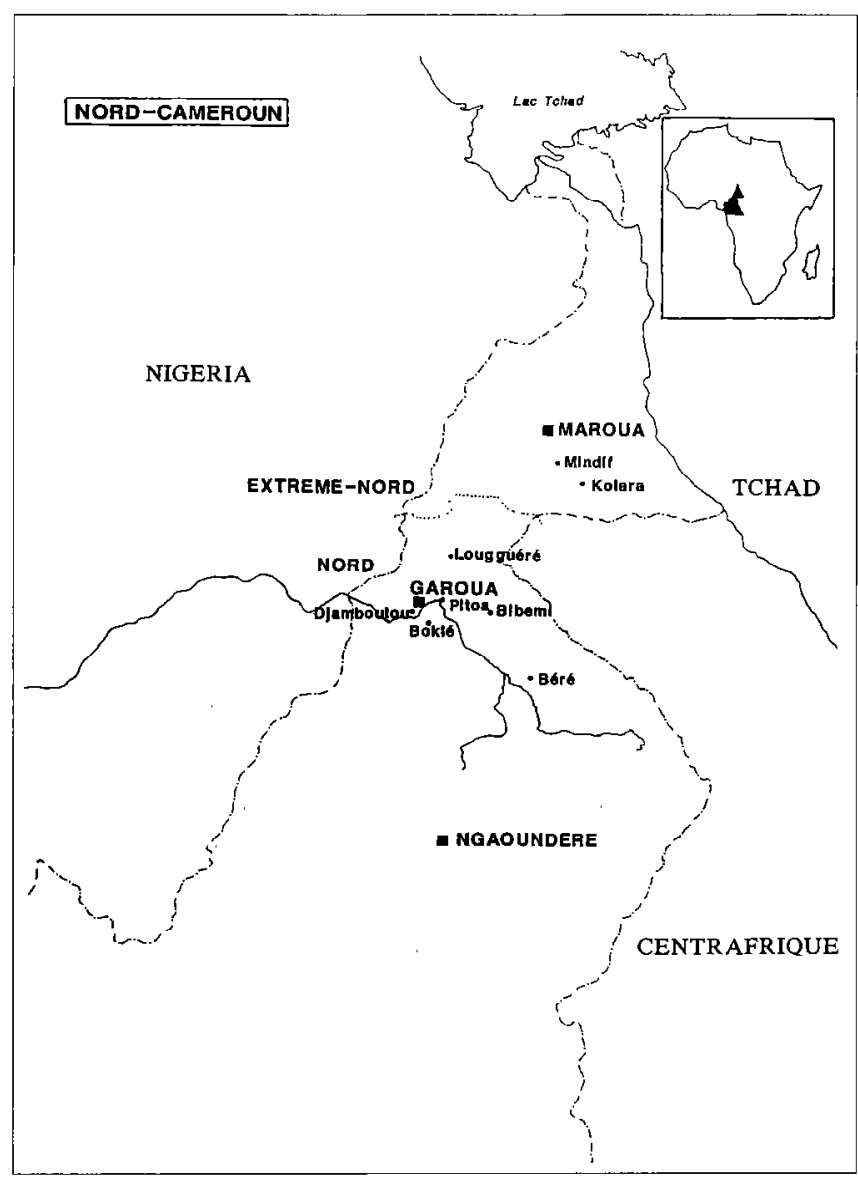

Carte 1: Carte du Nord-Cameroun.

Les élevages sont orientés vers la production de lait, de bœufs de labour, ou la capitalisation. La station zootechnique de Lougguéré diffuse des reproducteurs Arabes Choa. Certains troupeaux pratiquent une transhumance de saison sèche sur de courtes distances. Les animaux reçoivent une complémentation protéique de saison sèche sous forme de tourteau de coton.

Les jeunes sont généralement attachés à la corde à veaux pendant toute la journée jusqu'à l'âge d'un mois. Leur alimentation est alors exclusivement lactée. De 1 à 3 mois, ils sont en liberté autour des campements et audelà de cet âge, ils partent au pâturage avec les adultes. Le sevrage survient à un âge variable, fonction de la lactation de la mère. Les jeunes ne reçoivent pas de traitement prophylactique particulier, sauf dans le troupeau de Lougguéré. Certains éleveurs ont recours aux traitements anthelminthiques ponctuels en cas de troubles digestifs avec mauvais état général. Ces interventions curatives atteignent un niveau relativement important dans un des troupeaux de Boklé (troupeau $n^{\circ} 5$ ).

De novembre 1989 à octobre 1991, 193 veaux ont fait l'objet d'un suivi coproscopique mensuel dès l'âge d'un mois jusqu'à leur sortie du troupeau ou la fin du suivi. Le mauvais état général ainsi que les troubles digestifs éventuellement rencontrés ont été enregistrés lors des prélèvements. Un total de 1447 prélèvements ont été traités.

\section{Analyses coprologiques}

La stabilisation des œufs et oocystes de parasites par le froid s'avérant parfois impraticable, les prélèvements étaient formolés sur le terrain avec une solution à $5 \mathrm{p}$. 100. Comme il était impossible de peser chaque prélèvement, le facteur de dilution des fèces par adjonction d'eau formolée était variable d'un prélèvement à l'autre.

Au laboratoire, 2 techniques ont été utilisées en parallèle sur chaque prélèvement : la technique de sédimentation et celle de McMaster, mais en partant, dans les deux cas, de sédiment de matière fécale et non de fèces fraîches, afin de supprimer la variation de l'effet de dilution dû à l'adjonction d'eau formolée.

Chaque prélèvement était homogénéisé en totalité dans de l'eau dans un mortier, tamisé et mis à sédimenter pendant une heure dans un verre à pied. Après élimination du surnageant, $5 \mathrm{~g}$ de sédiment étaient alors mélangés à une solution saturée de $\mathrm{NaCl}$ (solution de Willis) pour examen en cellule de McMaster, et 5 autres grammes de sédiment étaient homogénéisés dans $3 \mathrm{ml}$ d'eau du robinet ; de cette dernière suspension, quelques gouttes étaient examinées entre lame et lamelle. En procédant ainsi, les comptages en cellules de McMaster ont donné des nombres d'œufs ou d'oocystes par gramme (OPG) de sédiment fécal. La lecture avec ces cellules a été effectuée sur un réseau par prélèvement, et en cas de valeur nulle sur ce réseau, la lecture était poursuivie sur le reste de la cellule.

Le facteur (constant) de dilution des prélèvements introduit au cours de ces manipulations représente la baisse de sensibilité de la méthode employée. II a été mesuré en comparant les nombres d'OPG en partant de fèces fraîches et de fèces formolées-sédimentées et a été trouvé égal à 2,8 .

\section{Analyse des données}

Etant donné que le nombre d'œufs ou d'oocystes de parasites émis dans les fèces est fluctuant et que la coproscopie est peu sensible, il a semblé préférable de calculer les prévalences en cumulant les résultats de plusieurs examens. Les prévalences des infestations et infections à Toxocara, strongles, Strongyloides et coccidies ont donc été calculées en considérant comme positifs tous les veaux des classes d'âge à risque qui ont fait l'objet d'au moins deux coproscopies, et qui ont présenté des œufs ou oocystes d'un groupe de parasites donné au moins une fois au cours du suivi.

L'interprétation des résultats obtenus en OPG de sédiment fécal a été faite en considérant les seuils de valeurs élevées suivants : $10000 / 2,8=3571$ pour les Toxocara 
TABLEAU I Caractéristiques géographiques et botaniques des terroirs de l'étude (d'après (35)).

\begin{tabular}{|c|c|c|c|c|}
\hline Localité & Latitude & Précipitations & Altitude & Végétation \\
\hline $\begin{array}{l}\text { Mindif } \\
\text { (Extrême- } \\
\text { Nord) }\end{array}$ & $10^{\circ} 26^{\prime} \mathrm{N}$ & $750 \mathrm{~mm}$ & $370 \mathrm{~m}$ & $\begin{array}{l}\text { Savane arborée à Burkea africana, Lophira lanceolata, Pericop- } \\
\text { sis laxiflora. } \\
\text { Strate herbacée à Schizachyrium sanguineum, Loudetia simplex, } \\
\text { L. togoensis, Andropogon gayanus, Hyparrhenia filipendula. }\end{array}$ \\
\hline $\begin{array}{l}\text { Kolara } \\
\text { (Extrême- } \\
\text { Nord) }\end{array}$ & $10^{\circ} 15^{\prime} \mathrm{N}$ & $800 \mathrm{~mm}$ & $378 \mathrm{~m}$ & $\begin{array}{l}\text { Steppe arbustive plus ou moins dégradée à Balanites } \\
\text { aegyptica, Guiera senegalensis, Bauhinia rufescens. } \\
\text { Strate herbacée à Schoenefeldia gracilis, L. togoensis, Era- } \\
\text { grostis gangetica. }\end{array}$ \\
\hline $\begin{array}{l}\text { Lougguéré } \\
\text { (Nord) }\end{array}$ & $9^{\circ} 56^{\prime} \mathrm{N}$ & $1000 \mathrm{~mm}$ & $450 m$ & $\begin{array}{l}\text { Steppe boisée à Boswellia dalzielii. } \\
\text { Strate herbacée à L. togoensis, Schizachyrium exile, Schoene- } \\
\text { feldia gracilis, Panicum laetum. }\end{array}$ \\
\hline $\begin{array}{l}\text { Pitoa } \\
\text { (Nord) }\end{array}$ & $9^{\circ} 23^{\prime} \mathrm{N}$ & $1000 \mathrm{~mm}$ & $190 \mathrm{~m}$ & $\begin{array}{l}\text { Savane arbustive de jachère à Terminalia laxiflora. } \\
\text { Strate herbacée à Andropogon pseudopricus, A. schirensis, } \\
\text { Diheteropogon amplectens, L. simplex. }\end{array}$ \\
\hline $\begin{array}{l}\text { Djamboutou } \\
\text { (Nord) }\end{array}$ & $9^{\circ} 18^{\prime} \mathrm{N}$ & $1000 \mathrm{~mm}$ & $190 \mathrm{~m}$ & $\begin{array}{l}\text { Savane arbustive de jachère à Terminalia laxiflora. } \\
\text { Strate herbacée à Andropogon pseudopricus, } A \text {. Schirensis, } \\
\text { Diheteropogon amplectens, L. simplex. }\end{array}$ \\
\hline $\begin{array}{l}\text { Boklé } \\
\text { (Nord) }\end{array}$ & $9^{\circ} 15^{\prime} \mathrm{N}$ & $1000 \mathrm{~mm}$ & $250 \mathrm{~m}$ & $\begin{array}{l}\text { Savane arborée à Burkea africana, Lophira lanceolata, Pericop- } \\
\text { sis laxiflora. } \\
\text { Strate herbacée à Schizachyrium sanguineum, Loudetia } \\
\text { simplex, L. togoensis, Andropogon gayanus, Hyparrhenia }\end{array}$ \\
\hline $\begin{array}{l}\text { Bibémi } \\
\text { (Nord) }\end{array}$ & $9^{\circ} 19^{\prime} \mathrm{N}$ & $900 \mathrm{~mm}$ & $250 \mathrm{~m}$ & $\begin{array}{l}\text { Savane arborée à Anogeissus leiocarpus, Stercullia setigera. } \\
\text { Strate herbacée à L. togoensis, Hyparrhenia bagirmica, Schi- } \\
\text { zachyrium exile. }\end{array}$ \\
\hline $\begin{array}{l}\text { Béré } \\
\text { (Nord) }\end{array}$ & $9^{\circ} 01 ' \mathrm{~N}$ & $1000 \mathrm{~mm}$ & $275 \mathrm{~m}$ & $\begin{array}{l}\text { Savane arbustive très claire à Pseudocedrela kotschyi, Termi- } \\
\text { nalia macroptera. } \\
\text { Strate herbacée à Loudetia simplex, Panicum anabaptistum, } \\
\text { Hyparrhenia rufa. }\end{array}$ \\
\hline
\end{tabular}

et les coccidies, et $1000 / 2,8=357$ pour les strongles et les Strongyloides. Les seuils de valeurs moyennes ont été fixés respectivement à $3000 / 2,8$ et $300 / 2,8$. Les seuils de 10000 et 1000 OPG couramment admis dans la littérature comme proches des seuils de pathogénicité $(9,12,27,31)$ ont été divisés par 2,8 afin de tenir compte de la baisse de sensibilité de notre méthode (facteur 2,8 ). Pour évaluer l'influence de différents facteurs sur l'importance du parasitisme, les variables suivantes ont été définies pour chaque parasite ou groupe de parasites:

- la présence ou l'absence d'œufs ou d'oocystes d'un type de parasite donné (modalités 0 ou 1) ;

- le nombre d'œufs ou d'oocystes par gramme de sédiment fécal (OPG) ;

- le caractère faible ou non, moyen ou non et élevé ou non du nombre d'OPG (modalités 0 ou 1 pour chacune de ces variables).
Les variables explicatives retenues ont été les suivantes:

- le sexe : 2 modalités ;

- la saison : 2 modalités, saison sèche du 1er novembre au 30 avril, saison des pluies du 1er mai au 31 octobre ;

- la province : 2 modalités, Nord et Extrême-Nord;

- la classe d'âge : 4 modalités, 0-3 mois, 3-6 mois, 6 mois-1 an et plus d'un an ;

- l'élevage : 17 modalités.

L'unité statistique a été l'animal-mois, sauf pour les calculs de prévalence où les données de plusieurs prélèvements ont été cumulées. L'analyse univariée des tableaux de contingence $2 \times 2$ a été réalisée à l'aide du test du $\chi 2$. L'analyse multivariée a été réalisée d'une part en effectuant une analyse factorielle des correspondances multiples (AFCM) suivie d'une classification ascendante hiérarchique (CAH) et; d'autre part, à l'aide 
d'une régression logistique multiple (RLM) sur les variables explicatives ayant une valeur de $p$ au $\chi^{2}$ inférieure à 0,20 . Par ailleurs, des analyses de variance ont été effectuées après transformation logarithmique des nombres d'OPG.

\section{RESULTATS}

\section{Importance des différentes parasitoses digestives}

\section{Toxocara vitulorum}

\section{Prévalences globales}

Cent quarante-huit veaux des 17 troupeaux ont subi au moins 2 prélèvements entre 0 et 6 mois, dont 1 au cours des 3 premiers mois (veaux cibles). On obtient les chiffres suivants:

- positifs au moins une fois : $39 \pm 8$ p. 100 ( $p<5$ p. 100) ; - veaux ayant donné au moins une fois un prélèvement fécal avec un nombre élevé d'OPG : $24 \pm 7$ p. 100, soit $60 \pm 12$ p. 100 des veaux positifs;

- veaux présentant au moins une fois une coproscopie positive avec diarrhée et/ou mauvais état général : $10 \pm 5$ p. 100 , soit $26+11$ p. 100 des veaux positifs.

Prévalences par élevage (tabl. II)

En regroupant les données des troupeaux de localités voisines, on obtient les prévalences suivantes:

- Boklé-Djamboutou-Pitoa : $53 \pm 12,8$ p. 100 (58 veaux) ;

- Béré-Bibémi : 51 p. 100 (37 veaux ; IC* : 33-67 p. 100);

- Kolara-Mindif : 7 p. 100 (30 veaux ; IC : 1-22 p. 100);

- Lougguéré : 26 p. 100 (23 veaux ; IC : 12-52 p. 100) ;

TABLEAU II Prévalence par élevage de la toxocarose (nombre de veaux positifs au moins une fois/nombre de veaux de 0 6 mois).

\begin{tabular}{|llll|}
\hline \multicolumn{2}{|c|}{ Nord } & & \multicolumn{2}{c|}{ Extrême-Nord } \\
\cline { 2 - 4 } Troupeau & $\begin{array}{c}\text { Veaux }+ \text { / } \\
\text { veaux suivis }\end{array}$ & Troupeau & $\begin{array}{c}\text { Veaux }+ \text { / } \\
\text { veaux suivis }\end{array}$ \\
\hline Béré 12 & $4 / 10$ & Kolara 9 & $1 / 8$ \\
Béré 13 & $9 / 11$ & Kolara 10 & $0 / 8$ \\
Bibémi 16 & $1 / 10$ & Mindif 8 & $0 / 9$ \\
Bibémi 17 & $5 / 6$ & Mindif 11 & $1 / 5$ \\
Boklé 1 & $5 / 7$ & & \\
Boklé 5 & $2 / 12$ & & \\
Boklé 7 & $6 / 8$ & & \\
Boklé 15 & $6 / 10$ & & \\
Boklé 19 & $4 / 6$ & & \\
Djamboutou 3 & $4 / 7$ & & \\
Djamboutou 4 & $3 / 4$ & & \\
Pitoa 2 & $1 / 4$ & & \\
Lougguéré 14 & $6 / 23$ & & \\
\hline
\end{tabular}

soit $47 \pm 9$ p. 100 de veaux positifs dans le Nord, et 7 p. 100 dans l'Extrême-Nord (IC : 1-22 p. 100). En fait, pour le Nord, il convient d'écarter les données de 2 élevages bénéficiant d'un bon appui sanitaire qui biaisent les résultats, ce qui porte la prévalence de la toxocarose à $58 \pm 10,6$ p. 100 dans cette province.

\section{Strongyloides}

\section{Prévalences globales}

Dans les classes d'âge à risque (0 à 12 mois), 75,5 \pm 6,7 p. 100 des 159 veaux ayant subi au moins deux coproscopies excrètent des œufs de Strongyloides au cours de leur carrière. Des infestations apparentes fortes ont été rencontrées au moins une fois chez $31 \pm 7$ p. 100 des veaux $(5,9 \pm 1,3$ p. 100 des prélèvements). Les manifestations cliniques qui accompagnent parfois ces fortes infestations apparentes ne sont pas dues spécifiquement à la strongyloïdose, les veaux excrétant alors simultanément des œufs de Toxocara. Les infestations patentes avant 9 jours ont été peu nombreuses : seulement 7 observations sur 245 prélèvements positifs.

\section{Différences entre provinces}

Malgré les effets province et élevage démontrés sur les nombres d'OPG, les prévalences de la strongyloïdose dans les deux provinces ne sont pas significativement différentes : respectivement $74,8 \pm 7,6$ et 78,1 p. 100 (IC : 64-93 p. 100) dans le Nord et l'Extrême-Nord. Ceci voudrait dire que les veaux ne sont pas plus fréquemment infestés dans le Nord que dans l'Extrême-Nord, mais qu'ils le sont de façon plus importante.

\section{Strongles}

Tous les bovins des classes d'âge concernées (plus de 6 mois) excrètent des œufs de strongles au moins une fois au cours de leur suivi. $35 \pm 9$ p. 100 des bovins de ces classes d'âge présentent des infestations apparentes fortes au moins une fois, et $55 \pm 9$ p. 100 des infestations apparentes moyennes. $6,8 \pm 2$ p. 100 des prélèvements de ces animaux ont révélé des nombres d'OPG élevés, et $15,1 \pm 2,7$ p. 100 des nombres d'OPG moyens. Les prévalences dans les deux provinces ne sont pas significativement différentes.

\section{Coccidies}

\section{Prévalences globales}

La prévalence de la coccidiose dans les classes d'âge à risque (idem Strongyloides) est globalement de 77,4 \pm 6,5 p. 100. Les infections fortes sont peu fréquentes : $7 \pm$ 4 p. 100 des veaux de 0 à 12 mois au moins une fois au

* Pour les petits effectifs, l'intervalle de confiance (IC) dissymétrique est précisé in extenso 
cours du suivi. $1 \pm 0,6$ p. 100 des prélèvements contient un grand nombre d'oocystes, et seuls 3 prélèvements ont révélé plus de $35700(100000 / 2,8)$ oocystes par gramme de sédiment fécal.

\section{Différences entre provinces}

Les prévalences dans le Nord et l'Extrême-Nord sont significativement différentes $(p<0,01)$ : respectivement $81,9 \pm$ 6,7 p. 100 et 59,4 p. 100 (IC : 44-79 p. 100) des veaux. Par ailleurs, étant donné l'effet province mis en évidence sur les nombres d'OPG et la fréquence de prélèvements positifs, il semble que les veaux soient plus souvent infestés dans le Nord que dans l'Extrême-Nord, et de façon plus importante; le fait que l'analyse de variance et la RLM n'aient pu révéler de différence significative pour la variable "OPG coccidies élevé" est certainement dû au faible nombre de prélèvements fortement positifs.

\section{Autres parasitoses digestives}

Les prélèvements contenant des œufs de trichures, Moniezia spp, paramphistomes et grandes douves se sont avérés très peu nombreux:

- Fasciola gigantica : 3 cas (1,5 \pm 1,5 p. 100 des prélèvements de la classe d'âge 4);

- Moniezia spp : 12 cas $(1,8 \pm 1$ p. 100 dans les classes d'âge 3 et 4) ;

- Trichuris spp : 14 cas $(2,1 \pm 1,1$ p. 100 dans les classes d'âge 3 et 4) ;

- Paramphistomes : 17 cas $(8,4 \pm 3,8$ p. 100 dans la classe d'âge 4).

\section{Origine des fluctuations des nombres d'OPG}

La figure 1 représente la projection de l'AFCM sur le plan défini par les axes 1 et 2, projection sur laquelle les variables illustratives sont bien représentées. Seules les variables explicatives ayant une contribution relative aux axes supérieure à la moyenne ont été indiquées. La figure 2 représente le dendrogramme de la $\mathrm{CAH}$ effectuée à partir de l'AFCM. Les résultats de la régression logistique multiple et des analyses de variance sont indiqués dans les tableaux III et IV respectivement.

\section{Toxocara vitulorum}

Les analyses statistiques montrent une influence très significative et prépondérante de l'âge sur la présence et le nombre d'œufs de Toxocara. La CAH montre qu'une partition en 7 groupes (correspondant à 61 p. 100 de l'inertie expliquée) regroupe les variables "classe d'âge 03 mois", "OPG Toxocara élevé" et "OPG Strongyloides élevé" (fig. 2). Dans la classe d'âge 0-3 mois, les taux de prélèvements positifs et fortement positifs sont respectivement de $22,6 \pm 4$ p. 100 et $11,6 \pm 3$ p. 100 , contre $1,1 \pm$ 0,6 p. 100 et 0,0 p. 100 dans les autres classes d'âge.

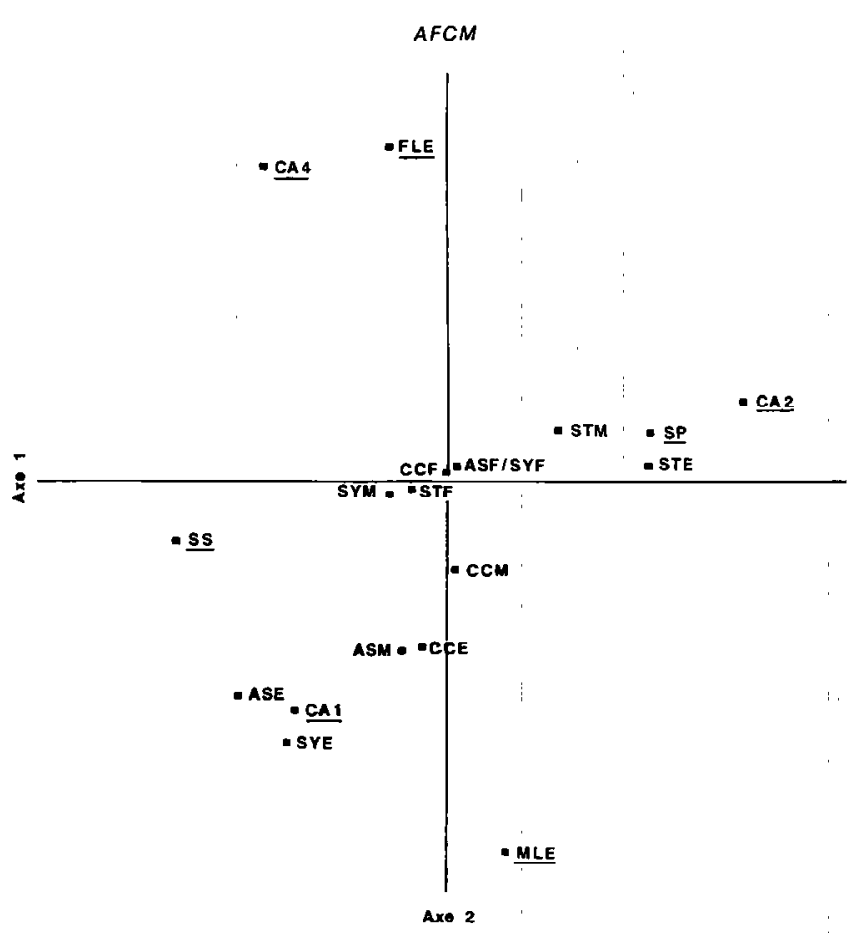

Figure 1 : Projection de l'analyse factorielle des correspondances multiples (AFCM) sur le plan défini par les axes 1 et 2. (Pourcentages d'inerties extraites par les axes $1,2,3,4$ et $5: 30,7 ; 25,7 ; 20,7 ; 16,1$; 6,8.)

Variables illustratives

$A S E=O P G$ Toxocara élevés $; A S M=O P G$ Toxocara moyens $; A S F=$ $O P G$ Toxocara faibles $; C C E=O P G$ coccidies élevés $C C M=O P G$ coccidies moyens ; $C C F=O P G$ coccidies faibles $; S T E=O P G$ strongles élevés ; $S T M=O P G$ strongles moyens ; $S T F=O P G$ strongles faibles ; $S Y E=O P G$ Strongyloides élevés; $S Y M=O P G$ Strongyloides moyens ; $S Y F=O P G$ Strongyloides faibles.

Variables explicatives

CAI = Classe d'âge 1 (0-3 mois) ; CA2 = Classe d'âge 2 (3-6 mois) CA4 = Classe d'âge $4(>12$ mois $) ; S P=$ Saison des pluies $; S S=$ Saison sèche $; F L E=$ Femelle $;$ MLE $=$ Mâle.

Les variables explicatives "Nord", "Extrême-Nord" et "Classe d'âge 3" ont été retirées de cette AFCM en raison de leurs faibles contributions relatives à l'inertie expliquée par les axes 1 et 2.

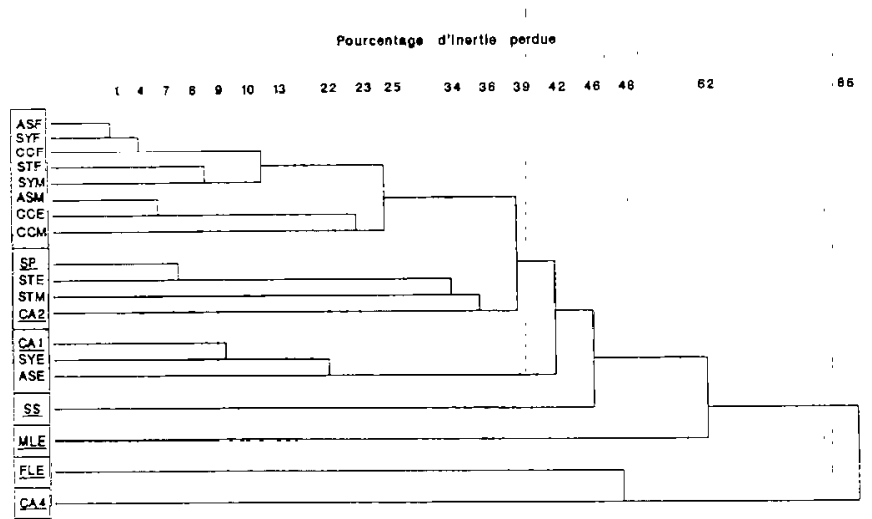

Figure 2 : Dendrogramme de la classification ascendante hiérarchique (CAH) effectuée à partir de l'analyse factorielle des correspondances multiples (AFCM) par la distance euclidienne et l'agrégation du saut minimum. Partition en 7 groupes. (Variables illustratives et explicatives : voir figure I) 
L'effet province est également important et les taux de prélèvements positifs et fortement positifs dans la classe d'âge 1 sont respectivement de $27,2 \pm 4,8$ p. 100 et 14,2 $\pm 3,7$ p. 100 dans le Nord, contre $5,6 \pm 4,7$ p. 100 et $2,2 \pm$ 3 p. 100 dans l'Extrême-Nord.

L'élevage influence significativement les pourcentages de prélèvements positifs et fortement positifs (tabl. III) ; la zone géographique pèse lourdement sur les fluctuations dues à cette variable. Les différences entre localités sont globalement significatives si l'on considère les prévalences de veaux cibles positifs $\left(\chi^{2}, 7 \mathrm{ddl}, \mathrm{p}<0,001\right)$. La différence entre la zone Boklé-Djamboutou-Pitoa et BéréBibémi n'est pas significative. Après regroupement des données de ces 5 localités, la différence entre BokléDjamboutou-Pitoa-Béré-Bibémi d'une part, et Kolara-Mindif d'autre part, est très significative $\left(\chi^{2}, 1 \mathrm{ddl}, p<0,001\right)$.

L'AFCM met également en évidence un effet saison sur la fréquence d'OPG élevés. Dans la classe d'âge 1, les taux de prélèvements positifs en saison sèche et en saison des pluies sont respectivement de $23,7 \pm 5,4$ et $21 \pm$ 5,9 p. 100 , la différence n'étant pas significative. Le test du $\chi^{2}$, de même que les analyses de variance et les RLM montrent que cet effet saison est significatif seulement sur les taux de prélèvements fortement positifs ; dans la classe d'âge $0-3$ mois, $15 \pm 4,5 p$. 100 des prélèvements ont des nombres élevés d'OPG en saison sèche contre $7,2 \pm 3,8$ p. 100 en saison des pluies $\left(\chi^{2}, 1 \mathrm{ddl}, p<0,02\right)$.

L'AFCM tend à montrer un certain effet du sexe sur les variables "OPG Toxocara élevé" et "OPG Toxocara moyen", mais il s'avère non significatif (tabl. III).

\section{Strongyloides}

Comme le montre la partition en 7 groupes réalisée par la $\mathrm{CAH}$ (figure 2), l'effet principal est représenté par l'âge. $40,4 \pm 4,7$ p. 100 des prélèvements sont positifs dans la classe d'âge 0-3 mois, contre 7,4 $\pm 1,6$ p. 100 dans l'ensemble des autres classes d'âge ; les infestations fortes sont rencontrées presque uniquement dans la classe d'âge $0-3$ mois $(16,4 \pm 3,5$ p. 100 des prélèvements dans cette classe d'âge).

L'influence de la province et de l'élevage sur les variables "OPG Strongyloides" et "OPG Strongyloides élevé" est également significative ; les fréquences de prélèvements positifs et fortement positifs pour la classe d'âge 0-3 mois sont respectivement de $31,1 \pm 9,6$ et $6,7 \pm 5,1$ p. 100 des prélèvements dans l'Extrême-Nord contre 42,9 $\pm 5,3$ et $19 \pm 4,2$ p. 100 dans le Nord.

Les tests effectués mettent également en évidence un effet sexe significatif sur la variable "OPG Strongyloides". Les mâles sont infestés plus fréquemment que les femelles : respectivement, $46,8 \pm 6,6$ et $33,5 \pm 6,5$ p. 100 $\left(\chi^{2}, 1\right.$ ddl, $\left.p<0,01\right)$.

\section{Strongles}

Les effets dus à l'âge et la saison sont les plus importants. La partition en 7 groupes réalisée par la $\mathrm{CAH}$ (fig. 2) regroupe les variables "saison des pluies", "classe d'âge 36 mois", "OPG strongles élevé" et "OPG strongles moyen". Les fréquences de prélèvements positifs entre 3 et 12 mois sont les plus fortes, surtout entre 6 et 12 mois : 56,9 $\pm 3,4$ p. 100 dans les classes d'âge 2 et 3 confondues, alors qu'elles sont respectivement de $18,3 \pm 3,7$ et $45 \pm$ 6,9 p. 100 dans les classes d'âge 1 et 4 .

La saison a une influence prépondérante sur les infestations fortes : elles sont rencontrées presque uniquement en saison des pluies $(10,7 \pm 2,1$ p. 100 des prélèvements, contre $1,3 \pm 0,9$ p. 100 en saison sèche), et surtout entre 6 et 12 mois. Le sexe n'intervient pas dans les fluctuations des variables "OPG strongles" et "OPG strongles élevé", y compris chez les animaux de plus de 12 mois.

Les analyses de variance et les RLM ne permettent pas de conclure à un effet significatif de la province. En revanche, le facteur élevage influence très significativement les variables "OPG strongles" et "OPG strongles élevé" ; peut-être y a-t-il un véritable effet "mode d'élevage" indépendant du facteur géographique.

\section{Coccidies}

L'effet principal est représenté par l'âge : les fréquences de prélèvements positifs dans les classes d'âge 1 et 2 confondues, 3 et 4 sont respectivement de 24,3: $\pm 3 p$. $100,32,2 \pm 4,3$ et $18,3 \pm 5,3$ p. 100 . La fréquence est donc la plus élevée dans la classe d'âge 6-12 mois. En revanche, les infections fortes sont rencontrées quasi exclusivement dans les classes d'âge 0-3 mois et 3-6 mois (respectivement $2,1 \pm 1,4$ et $0,8 \pm 1$ p. 100 des prélèvements).

La province et l'élevage ont également une influence très significative sur la variable "OPG coccidies". Les proportions de prélèvements positifs dans le Nord et l'ExtrêmeNord (respectivement $27,3 \pm 2,6$ et $21 \pm 4,5$ p. 100) sont elles aussi significativement différentes $\left(\chi^{2}, 1 \mathrm{ddl}\right.$, $p<0,03)$. En revanche, les tests ne permettent pas de mettre en évidence d'influence significative' sur la variable "OPG coccidies élevé".

Comme pour les Toxocara et les Strongyloides, l'AFCM montre une relative proximité du sexe mâle avec la modalité positive de la variable "OPG coccidies élevé", mais l'analyse de variance ne permet pas de mettre en évidence de différence significative entre les deux sexes.

\section{DISCUSSION}

La toxocarose des veaux doit particulièrement retenir l'attention, principalement dans la province du Nord où elle atteint 58 p. 100 des veaux de 0-6 mois. Pour l'Extrême-Nord, le chiffre de 7 p. 100 de veaux positifs doit être comparé aux 32 p. 100 rapportés par GRABER et PERROTIN pour la région de Maroua (12). On doit reconnaître que si la représentativité de l'échantillon est satis- 
TABLEAU III Comparaison des moyennes d'OPG : degrés de significativité des tests "F"' des analyses de variance.

\begin{tabular}{|c|c|c|c|c|c|c|c|c|}
\hline & $\begin{array}{c}\text { Toxocara } \\
\text { (log } \\
\text { OPG) }\end{array}$ & $\begin{array}{l}\text { OPG } \\
\text { Toxocara } \\
\text { élevé }\end{array}$ & $\begin{array}{c}\text { Strongles } \\
\text { (log } \\
\text { OPG) }\end{array}$ & $\begin{array}{c}\text { OPG } \\
\text { Strongles } \\
\text { élevé }\end{array}$ & $\begin{array}{c}\text { Strongyloides } \\
\text { (log } \\
\text { OPG) }\end{array}$ & $\begin{array}{c}\text { OPG } \\
\text { Strongyloides } \\
\text { élevé }\end{array}$ & $\begin{array}{c}\text { Coccidie } \\
\text { (log } \\
\text { OPG) }\end{array}$ & $\begin{array}{c}\text { OPG } \\
\text { coccidies } \\
\text { élevé }\end{array}$ \\
\hline $\begin{array}{l}\text { Classe d'âge } \\
\text { Province } \\
\text { Saison } \\
\text { Sexe }\end{array}$ & $\begin{aligned} &< 0,001 \\
&<0,001 \\
& \text { NS } \\
& \text { NS }\end{aligned}$ & $\begin{array}{c}<0,001 \\
0,002 \\
0,011 \\
\text { NS }\end{array}$ & $\begin{aligned} &< 0,001 \\
& \text { NS } \\
&<0,001 \\
& \\
& \text { NS }\end{aligned}$ & $\begin{aligned} & 0,007 \\
& \text { NS } \\
< & 0,001 \\
& \text { NS }\end{aligned}$ & $\begin{array}{c}<0,001 \\
0,007 \\
\text { NS } \\
0,047\end{array}$ & $\begin{array}{l}0,001 \\
0,003 \\
0,037 \\
\text { NS }\end{array}$ & $\begin{array}{l}0,002 \\
0,012 \\
\text { NS } \\
\text { NS }\end{array}$ & $\begin{array}{l}0,012 \\
\text { NS } \\
\text { NS } \\
\text { NS }\end{array}$ \\
\hline Elevage & $<0,001$ & 0,001 & $<0,001$ & $<0,001$ & 0,004 & $<0,001$ & 0,001 & NS \\
\hline
\end{tabular}

OPG : nombre d'ceufs ou d'oocystes par gramme de sédiment fécal. NS : non significatif.

TABLEAU IV Analyse de l'influence des marqueurs de risque sur les valeurs d'OPG par régressions logistiques multiples.

\begin{tabular}{|c|c|c|c|c|c|c|c|c|c|}
\hline & & $\begin{array}{l}\text { Présence } \\
\text { Toxocara }\end{array}$ & $\begin{array}{c}\text { OPG } \\
\text { Toxocara } \\
\text { élevé }\end{array}$ & $\begin{array}{l}\text { Présence } \\
\text { strongles }\end{array}$ & $\begin{array}{c}\text { OPG } \\
\text { strongles } \\
\text { élevé }\end{array}$ & $\begin{array}{c}\text { Présence } \\
\text { Strongyloides }\end{array}$ & $\begin{array}{c}\text { OPG } \\
\text { Strongyloides } \\
\text { élevé }\end{array}$ & $\begin{array}{l}\text { Présence } \\
\text { coccidies }\end{array}$ & $\begin{array}{c}\text { OPG } \\
\text { coccidies } \\
\text { élevé }\end{array}$ \\
\hline $\begin{array}{l}\text { Province } \\
\text { du Nord }\end{array}$ & $\begin{array}{l}\text { DS } \\
\text { RRA }\end{array}$ & $\begin{array}{l}0,0001 \\
5,72\end{array}$ & $\begin{array}{l}0,0051 \\
7,83\end{array}$ & $\begin{array}{l}0,3941 \\
1,13\end{array}$ & $\begin{array}{l}0,5772 \\
1,16\end{array}$ & $\begin{array}{l}0,0197 \\
1,6\end{array}$ & $\begin{array}{l}0,0036 \\
3,65\end{array}$ & $\begin{array}{l}0,028 \\
1,4078\end{array}$ & $\begin{array}{l}0,2506 \\
3,32\end{array}$ \\
\hline $\begin{array}{l}\text { Sexe } \\
\text { mâle }\end{array}$ & $\begin{array}{l}\text { DS } \\
\text { RRA }\end{array}$ & - & - & - & - & $\begin{array}{l}0,0345 \\
1,39\end{array}$ & $\begin{array}{l}0,15 \\
1,45\end{array}$ & 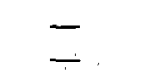 & - \\
\hline $\begin{array}{c}\text { Saison } \\
\text { sèche*/pluies }\end{array}$ & $\begin{array}{l}\text { DS } \\
\text { RRA }\end{array}$ & $\begin{array}{l}0,8899^{*} \\
1,03^{*}\end{array}$ & $\begin{array}{l}0,009^{*} \\
2,45^{\star}\end{array}$ & $\begin{array}{c}<0,0001^{* *} \\
4,01^{* \star}\end{array}$ & $\begin{array}{c}<0,0001^{* *} \\
9,07^{* *}\end{array}$ & $\begin{array}{l}0,9090^{*} \\
1,02^{*}\end{array}$ & $\begin{array}{c}0,0447^{*} \\
0,58^{*}\end{array}$ & - & $\begin{array}{l}0,9579^{\star \star} \\
1,03^{\star \star}\end{array}$ \\
\hline $\begin{array}{c}\text { Classe d'âge } \\
0-3 \text { mois }\end{array}$ & $\begin{array}{l}\text { DS } \\
\text { RRA }\end{array}$ & $\begin{array}{l}<0,0001 \\
27,34\end{array}$ & $\begin{array}{l}\S \\
\S\end{array}$ & - & - & $\begin{array}{c}<0,0001 \\
8,39\end{array}$ & $\begin{array}{c}<0,0001 \\
45,25\end{array}$ & - & $\begin{array}{c}0,0118 \\
14,32\end{array}$ \\
\hline $\begin{array}{c}\text { Classe d'âge } \\
3-6 \text { mois }\end{array}$ & $\begin{array}{l}\text { DS } \\
\text { RRA }\end{array}$ & - & - & - & - & - & - & $\begin{array}{l}0,6461 \\
0,93\end{array}$ & $\begin{array}{l}0,143 \\
5,55\end{array}$ \\
\hline $\begin{array}{l}\text { Classe d'âge } \\
6-12 \text { mois }\end{array}$ & $\begin{array}{l}\text { DS } \\
\text { RRA }\end{array}$ & - & - & $\begin{array}{c}<0,0001 \\
4,17\end{array}$ & $\begin{array}{l}0,0446 \\
1,57\end{array}$ & - & - & $\begin{array}{l}0,0184 \\
1,43\end{array}$ & - \\
\hline $\begin{array}{c}\text { Classe d'âge } \\
>12 \text { mois }\end{array}$ & $\begin{array}{l}\text { DS } \\
\text { RRA }\end{array}$ & - & - & $\begin{array}{c}<0,0001 \\
2,29\end{array}$ & $\begin{array}{l}0,1268 \\
0,48\end{array}$ & - & - & $\begin{array}{l}0,0688 \\
0,68\end{array}$ & - \\
\hline
\end{tabular}

RRA = risque relatif ajusté.

$\S$ : risques relatifs très élevés et non significatifs en raison d'effectifs nuls dans les tableaux de contingence. DS : degré de significativité.

faisante pour le Nord, elle est passable pour l'ExtrêmeNord : le nombre de veaux suivis y est faible et les troupeaux dont ils sont issus appartiennent à un seul département de cette province (département de Mayo Kani).

L'influence significative de la saison sur la fréquence de prélèvements ayant des valeurs élevées d'OPG de Toxocara est surprenante. A contrario, les fréquences de prélèvements positifs en saison sèche et en saison des pluies ne sont pas significativement différentes. Le fait que les analyses de variance ne permettent pas de mettre en évidence un effet "saison" sur la moyenne des nombres d'OPG est dû à la grande proportion de prélève- ments négatifs. La saison sèche n'a donc pas d'effet sur la prévalence mais semble, soit augmenter la ponte des Toxocara femelles, soit favoriser les infestations massives ; cette dernière hypothèse paraît plus vraisemblable. En aggravant l'immunodépression maternelle, les facteurs de stress liés à la saison sèche sont peut-être à l'origine d'une augmentation du nombre de larves parvenant au veau.

Etant donné la forte prévalence des Toxocara dans le Nord et le pouvoir pathogène important qu'on leur attribue habituellement, la rentabilité d'une lutte à coût minimal contre la toxocarose dans cette province nous 
semble probable. II y aurait lieu de comparer une vermifugation systématique simple des veaux à un mois $(4,12)$, une double vermifugation à un et trois mois (33) et un simple traitement des animaux déclarés malades, avec un benzimidazole ou un imidazothiazole bon marché.

La strongyloïdose est fréquente et souvent associée à la toxocarose. Contrairement à ce qui a été observé au Nigeria $(17,18)$, les cas d'infestations patentes avant 9 jours ont été peu nombreux (7 prélèvements), ce qui va dans le sens d'une période prépatente de durée classique et d'une transmission essentiellement post-natale chez les veaux, comme cela a été récemment réaffirmé (26). L'infestation apparente plus fréquente des mâles est une donnée originale qui demanderait à être confirmée. Cette liaison mâles/infestations fréquentes pourrait s'expliquer par le fait que l'éleveur accorderait plus de soins aux femelles. Cette dernière hypothèse semble confortée par certaines observations de terrain (NJOYA, non publié).

Une vermifugation systématique des veaux contre la toxocarose permettrait de lutter simultanément contre la strongyloïdose, à condition de prendre en compte l'activité faible ou moyenne des imidazothiazoles et de certains benzimidazoles contre les Strongyloides $(1,33)$. Le plus important serait sans doute de ne pas maintenir les jeunes veaux sur des endroits perpétuellement humides et souillés d'excréments, ce qui permettrait d'éviter les infestations transcutanées.

Les strongyloses digestives présentent des caractéristiques épidémiologiques semblables à celles décrites dans la littérature pour les élevages extensifs en zone tropicale $(2,3,5,12,25,31)$ : très forte prévalence, niveau moyen d'infestation apparente faible, influence 'significative des facteurs âge et saison. II n'a pas été retrouvé, comme en Guadeloupe, d'effet sexe (23), pas plus sur les animaux de plus d'un an que sur les autres.

II est très aléatoire d'identifier des mesures prophylactiques optimales à partir de simples données coproscopiques. Bien que les niveaux d'infestations apparentes étaient souvent peu élevés, plusieurs études ont démontré les effets négatifs des strongyloses subcliniques sur l'appétit, la digestibilité des protéines, l'utilisation de l'énergie digestible ainsi que les métabolismes phosphocalcique et protéique $(12,22,25,29)$.

En Afrique intertropicale, l'application de programmes de prophylaxie basés sur des vermifugations systématiques des bovins ou des petits ruminants ne fait pas l'unanimité, la rentabilité de ces opérations n'étant pas évidente $(4,15,16,23,30)$. II semblerait que les vermifugations systématiques n'augmentent significativement la vitesse de croissance que si les troupeaux ne sont pas maintenus sur des pâturages infestés $(7,30)$ ou si le rythme des traitements est élevé (4), c'est-à-dire lorsqu'il y a peu ou pas de réinfestations. Enfin, les variations climatiques inter-annuelles peuvent conduire à des modifications épizootiologiques $(10,13,15,16)$ qui peuvent influer sur la rentabilité économique d'un programme de prophylaxie.
Malgré une certaine controverse, il est probable que des traitements fréquents n'auraient pas d'intérêt économique $(19,24,25)$ et qu'ils augmenteraient le risque de résistance aux anthelminthiques $(1,14,30,34)$ dont des cas ont déjà été signalés au Cameroun et au Nigeria (25).

Les faciès parasitaires des bovins du Nord-Cameroun (6, 11) sont classiques pour l'Afrique centrale $(2,3,11,12$, $27,28,31$ ) ; ils sont dominés par des espèces réputées pathogènes (Haemonchus contortus, $H$. similis, Cooperia punctata, C. pectinata, Oesophagostomum radiatum), qui sont vraisemblablement responsables de baisses de performances des élevages. Dans la mesure où 35 p. 100 des bovins de plus de 6 mois ont présenté au moins une fois des nombres d'OPG élevés, il est possible qu'un programme de prophylaxie simple soit économiquement rentable. Mais en attendant que des essais de terrain aient permis de conclure sur la rentabilité des programmes de prophylaxie classiquement proposés $(12,19,24,25,31)$, il semble plus raisonnable, pour les bouvillons, de s'en tenir à des traitements curatifs chaque fois qu'une strongylose-maladie est envisagée.

La coccidiose est très répandue, et le niveau d'infection est rarement élevé. Elle ne paraît donc pas menaçante en élevage traditionnel, contrairement à ce qui a pu être observé dans certaines régions d'Afrique intertropicale (32) ; la situation du Nord-Cameroun vis-à-vis de cette maladie semble donc conforme au cas général en Afrique, caractérisé par une pathologie essentiellement restreinte aux élevages confinés et aux animaux ayant subi des stress importants. La prévalence et le niveau moyen d'infection sont plus élevés dans le Nord que dans l'Extrême-Nord, alors qu'au Sénégal la fréquence de cas cliniques ou subcliniques est d'autant plus élevée que le climat est aride (32). Actuellement, aucune prophylaxie anti-coccidienne systématique n'est à envisager en milieu paysan au Nord-Cameroun.

La rareté des Trichuris, Moniezia, Fasciola, et paramphistomes doit être discutée en considérant que la méthode coproscopique utilisée ici est peu sensible vis-à-vis de ces helminthes et, pour la fasciolose et la paramphistomose, que les données obtenues sur des animaux de plus de 18 mois ont été peu nombreuses (42 prélèvements). Les données d'abattoirs recueillies lors de précédentes études indiquent des prévalences allant jusqu'à 86 p. 100 pour la fasciolose et 58 p. 100 pour la paramphistomose à l'abattoir de Garoua (6).

\section{CONCLUSION}

La toxocarose apparaît comme la principale parasitose digestive des veaux de 0-3 mois dans le Nord. La strongyloïdose lui est souvent associée, mais ses effets pathologiques sont réputés moins graves. La lutte contre la strongyloïdose doit commencer par l'application de mesures sanitaires simples permettant d'éviter les infestations transcutanées. Dans le Nord, des traitements sys- 
tématiques dirigés principalement contre la toxocarose devraient être rentables, pour peu qu'ils soient réalisés avec des produits adaptés. II conviendrait néanmoins de le vérifier en testant plusieurs protocoles, notamment le traitement des veaux à un mois avec un vermifuge classique.

Le problème des strongyloses est plus complexe, et il est difficile dans l'immédiat de préconiser une conduite à tenir. Etant donné les prévalences d'infestations moyennes et fortes rencontrées, il est probable que les strongyloses entraînent des baisses de performances notables. II serait souhaitable de tester plusieurs programmes de vermifugation systématiques des bouvillons, en prenant en compte tous les paramètres de performance d'un troupeau, afin d'en mesurer précisément la rentabilité.

\section{REMERCIEMENTS}

Ce travail a été financé par l'Institut de Recherches zootechniques et vétérinaires et le projet IRA-SEB III/Elevage, Cameroun.

\section{BIBLIOGRAPHIE}

1. BARRAGRY (T.). Anthelmintics - review Part II. N. Z. vet. J., 1984, 32: 191-199.

2. CHARTIER (C.). Les dominantes du parasitisme helminthique chez les bovins en Ituri (Haut-Zaïre). I. La faune helminthique. Revue Élev. Méd. vét. Pays trop., 1990, 43 (1) : 75-84.

3. CHARTIER (C.), BUSHU (M.), ANICAN (U.). Les dominantes du parasitisme helminthique chez les bovins en Ituri (Haut-Zaïre). II. Les associations parasitaires. Revue Élev. Méd. vét. Pays trop., 1990, 43 (4) : 491-497.

4. CHARTIER (C.), BUSHU (M.), CABARET (J.). Effets de traitements anthelminthiques sur les gains de poids et les mortalités des jeunes bovins en Ituri (Nord-Est Zaïre). Revue méd. vét., 1991, 142 (1) : 57-63.

5. CHARTIER (C.), BUSHU (M.), KAMWENGA, (D.). Les dominantes du parasitisme helminthique chez les bovins en Ituri (Haut-Zaïre). III. Répartition géographique et prévalence des principaux helminthes. Revue Élev. Méd. vét. Pays trop., 1991, 44 (1) : 61-68.

6. CHOLLET (J.-Y.). Données épidémiologiques disponibles sur les parasites d'intérêt vétérinaire. In : Elevage et potentialités pastorales sahéliennes. Synthèses cartographiques. Cameroun-Nord. Wageningen, CTA. Maisons-Alfort, CIRAD-IEMVT eds, 1991. p. 22.

7. DOCKES (C.), FAUGERE (B.), FAUGERE (O.), MERLIN (P.), PERROT (C.). L'élevage traditionnel des petits ruminants dans la zone de Kolda. Programme pathologie et productivitè des petits ruminants en milieu traditionnel. Dakar, ISRA, Maisons-Alfort, CIRAD-IEMVT, mars 1988. (Document de travail $n^{\circ} 1$ )

8. ESTERE (P.). Epidémiologie des parasitoses digestives des bovins en Guadeloupe. Revue Élev. Méd. vét. Pays Trop., 1985, 38 (1) : 54-63.

9. EUZEBY (J.). Diagnostic expérimental des helminthoses animales. Tome 1. Paris, Informations techniques des Services vétérinaires, Ministère de l'Agriculture, $1981.349 \mathrm{p}$.

10. FABIYI (J. P.), OLUYEDE (D. A.), NEGEDU (J. O.). Late dry season outbreak of clinical haemonchosis and cooperiasis in cattle of northern Nigeria. Vet. Rec., 1979, 105: 399-400.
11. GRABER (M.), FERNAGUT (R.), OUMATIE (O.). Helminthes des zébus adultes de la région de Maroua (Nord-Cameroun). Revue Élev. Méd. vét. Pays trop., 1966, 19, (2) : 149-162.

12. GRABER (M.), PERROTIN (C.). Helminthes et helminthoses des ruminants domestiques d'Afrique tropicale. Maisons-Alfort, Editions du Point vétérinaire, $1983.378 \mathrm{p}$.

13. GRABER (M.), TAGER-KAGAN (P.). Inhibition des larves de Cooperia punctata et de Cooperia pectinata chez le zébu nigérien. Revue Élev. Méd. vét. Pays trop., 1975, 28 (2) : 137-142.

14. GRUNER (L.). Contrôle des strongyloses digestives des petits ruminlants aux Antilles françaises : développenent de résistances aux benzimidazoles et intérêt d'une gestion raisonnée des pâturages. Revue Élev. Méd. vét. Pays trop., 1985, 38 (4) : 386-393.

15. HUBERT (J.), KERBOEUF (D.), GRUNER (L.). Study of gastrointestinal strongylosis in a sheep flock on permanent pasture. 1. Sheep parasitism in 1977. Annls Rech. vét, 1979,10 (4): 503-518

16. HUBERT (J.), KERBOEUF (D.). Study of gastro-intestinal strongylosis in a sheep flock on permanent pasture. 2. Sheep parasitism in 19781979. Annls Rech. vét., 1985, 16 (1): 29-39.

17. IKEME (M. M.). Strongyloides papillosus and Neoascaris vitulorum naturally acquired mixed infestations of calves in the Plateau area of northern Nigeria and the treatment given. Bull. epizoot. Dis. Afr., 1970, 18: $339-345$.

18. NWAORGU (O. C.), ONYALI (I. O.). Strongyloides papillosus: prenatal and transmammary infection in ewes. Revue Élev. Méd. vét. Pays trop., 1990, 43 (4): 503-504.

19. OMEKE (B. C. O.). Evaluation of three strategic prohylactic programmes against helminthiasis of traditionnally managed west african dwarf sheep and goats in Nigeria. Br. vet. J., 1988, 144 (6): 590-595.

20. PARENT (R.), ALOGNINOUWA (TH.). Amélioration de la productivité de l'élevage en zone tropicale, traitement systématique des vaches gestantes à l'ivermectine dans les mois précédant la mise bas. Revue Elev. Méd. vét. Pays trop., 1984,37 (3) : 341-354.

21. PARENT (R.), SAMB (F.). Utilisation de l'ivermectine en milieu tropical, étude sur de jeunes bovins à l'embouche. Revue Élev. Méd. vét. Pays trop., 1984, 135 (3) : 131-134.

22. RANDALL (R.W.), GIBBS (H.C.). Effects of subclinical gastrointestinal helminthiasis on digestion and energy metabolism in calves. Am. J. vet. Res., 1981, 42 (10): 1730-1734.

23. SALAS (M.), SHEIKBOUDOU (C.). Le parasitisme digestif dans lès systèmes d'élevage bovin traditionnel en Guadeloupe. I. Enquête globale. Revue Élev. Méd. vét. Pays trop., 1988, 41 (2) : 171-180.

24. SALAS (M.), SHEIKBOUDOU (C.). Le parasitisme digestif dans lés systèmes d'élevage bovin traditionnel en Guadeloupe. II. Suivi de l'infestation parmi plusieurs groupes de veaux. Revue Élev. Méd. vét. Pays trop., $1988,41(4): 367-373$

25. SCHILLHORN VAN VEEN (T.W.), OGUNSUSI (R.A.). Epidémiologie et contrôle de l'helminthiase gastro-intestinale chez les moutons au Nigeria. In : XLVIe Session Générale du Comité de l'OIE, Paris, 22-27 mai 1978. Paris, OIE. 12 p.(Rapport $\mathrm{n}^{\circ} 106$ bis)

26. SHOOP (W.L.). Vertical transmission of helminths: hypobiosis and amphiparatenesis. Parasitol. Today, 1991, 7 (2): 51-54.

27. SOULSBY (E.J.L.). Helminths, arthropods and protozoa of domesticated animals. London, Baillière Tindall, 1982.809 p

28. STEWART (T.B.), GASBARRE (L.C.). The veterinary importance of nodular worms (Oesophagostomum spp). Parasitol. Today, 1989, 5 (7): 209-213.

29. SYKES (A. R.). The effect of subclinical parasitism in sheep. Vet. Rec., 1978, Jan. 14: 32-34. 


\section{J.-Y. Chollet A. Martrenchar D. Bouchel A. Njoya}

30. THYS (E.), VERCRUYSSE (J.). Est-il encore opportun de préconise la vermifugation systématique des petits ruminants d'Afrique sahélo-soudanienne contre les nématodes gastro-intestinaux ? Revue Elev. Méd. vét. Pays trop., 1990, 43 (2) : 187-191.

31. TRONCY (P. M.), ITARD (J.), MOREL (P. C.). Précis de parasitologie vétérinaire tropicale. Paris, Ministère de la Coopération et du Développement, $1981.717 \mathrm{p}$

32. VASSILIADES (G.). La coccidiose intestinale des ruminants domestiques au Sénégal, épidémiologie, répartition géographique, importance économique. Revue Elev. Méd. vét. Pays trop., 1969, 22 (1) : 47-53.
33. VASSILIADES (G.). Note techmique sur l'utilisation des anthelnninthiques dans la lutte contre les helminthoses du bétail au Sénégal. Dakar, ISRA, juillet 1980. $12 \mathrm{p}$.

34. WALLER (P.J.). Resistance in nematodes parasites of livestock to the benzimidazole anthelmintics. Parasitol. Today, 1990, 6 (4): 127-129.

35. ZBOROWSKI (I. de), GASTON (A.). Carte des potentialités pastorales du Nord-Cameroun. In : Élevage et potentialités pastorales sahéliennes. Synthèses cartographiques. Cameroun-nord. Wageningen, CTA, Maisons-Alfort, CIRAD-IEMVT, 1991. p. 6-8.
CHOLLET (J.-Y.), MARTRENCHAR (A.), BOUCHEL (D.), NJOYA (A.). Epidemiology of gastro-intestinal parasite infections of young cattle in Northern Cameroon. Revue Elev. Méd. vét. Pays trop., 1994, 47 (4): 365-374

Studies of gastro-intestinal parasites of zebu calves were carried out in traditional herds in Northern Cameroon through monthly faecal analysis in 17 herds for a period of two years. Toxocarosis appeared to be the most important parasitic infection in the North province where its prevalence reached $58 \%$ in calves aged 0-6 months; $60 \%$ of the infested calves passed large numbers of eggs at least once. Samples revealing high egg counts were more frequent in the dry season. Deworming calves aged one month with a cheap anthelmintic against Toxocara is likely to be economically profitable in the North. Seventy-five and a half per cent of the calves 0-12 months old were infested with Strongyloides; high counts of Strongyloides eggs were registered at least once for $31 \%$ of these calves. As for toxocarosis, calves seemed to be more often and more heavily infested with Strongyloides in the North than in the Far North province. Strongyloidosis was apparently of low clinical importance, with the rare clinical manifestations accompanied by toxocarosis. The importance of digestive tract strongylosis was difficult to evaluate. Every steer was affected at one stage of its life in a similar manner in the two provinces; $6.8 \%$ of samples showed high egg counts and $35 \%$ of the steers aged over six months passed large numbers of eggs at least once. These results did not permit a priori recommendation of a systematic deworming programme against strongyles; instead, several less intensive deworming programmes have to be tested in order to determine their economic profitability. Coccidia were found in $77.4 \%$ of calves aged 0-12 months, with heavier and more frequent infections in the North. However, infections of high intensity were generally rare. Trichuris, Moniezia, Fasciola and paramphistomes were rarely found. Concerning nematodosis, curative treatments and cost-profit studies of deworming programmes should be aimed at toxocarosis in calves aged 0-3 months in the North, and strongylosis in steers aged 6-12 months, in both provinces.

Key words: Cattle - Zebu cattle - Helminthosis - Coccidiosis - Strongylidae - Trichostrongylidae - Strongyloides - Toxocara vitulorum - Epidemiology - Disease control - Cost-profit analysis - Traditional farming Cameroon.
CHOLLET (J.-Y.), MARTRENCHAR (A.), BOUCHEL (D.), NJOYA (A.). Epidemiología de las parasitosis digestivas del bovino joven en el norte de Camerún. Revue Elev. Méd. vét. Pays trop., 1994, 47 (4) : $365-$ 374

Durante 2 años se estudiaron las parasitosis digestivas del joven hovino cebú en medio tradicional en el norte de Camerún, gracias al seguimiento coproscópico mensual de 17 hatos. La toxocarosis es la más importante en la provincia del Norte, donde la prevalencia alcanza 58 p. 100 entre 0 y 6 meses; 60 p. 100 de los terneros positivos excretan, al menos una vez, grandes cantidades de huevos de Toxocara. La frecuencia de las muestras, en las cuáles se observa gran número de huevos, debe ser superior durante la estación seca. La desparasitación de los terneros de un mes contra Toxocara, con un producto barato, podría ser rentable en el Norte. La estrongiloidosis ataca 75,5 p. 100 de los terneros de 0 a 12 meses. EI número de huevos de Strongyloides es alto al menos una vez, en 31 p. 100 de estos terneros. Al igual que con Toxocara, los terneros son parasitados más a menudo y en mayor cantidad en el Norte, que en el Extremo-Norte. La importancia clínica de la estrongiloidosis parece mínima y los raros problemas observados se confunden con aquellos provocados por la toxocarosís. La importancia de los estróngilos digestivos es difícil de evaluar: todos los toretes se encuentran afectados en algún momento y de manera idéntica en las dos provincias. El número de huevos de estróngilos en las heces de los bovinos de más de seis meses, es elevado en 6,8 p. 100 de las muestras y al menos en una ocasión en el 35 p. 100 de estos animales. Los resultados no permiten preconizar a priori un programa sistemático de desparasitación contra los estróngilos, siendo necesario probar varios programas simples para determinar la rentabilidad. En 77,4 p. 100 de los terneros de 0 a 12 meses se encontraron coccidios, con infecciones más frecuentes y más importantes en el Norte, sin embargo, éstas fueron rara vez fuertes. Los Trichuris, Moniezia, Fasciola y paramfistomas fueron observados unicamente en un pequeño número de casos. En cuanto a los nemátodos, las intervenciones curativas y los estudios de costo y beneficio de los programas de fumigación deben ser enfocados hacia la toxocarosis para los terneros de 0 a 3 meses en el Norte y hacia los estróngilos, en los jóvenes de 6 a 12 meses de las dos provincias.

Palabras clave : Bovino - Cebú - Helmintosis - Coccidiosis - Strongylidae - Trichostrongylidae - Strongyloides - Toxocara vitulorum - Epidemiología - Control de enfermedades - Análisis de costo y beneficio Crianza tradicional - Camerún. 